\title{
Development and preliminary validation of the focused analgesia selection test to identify accurate pain reporters
}

This article was published in the following Dove Press journal:

Journal of Pain Research

9 February 2017

Number of times this article has been viewed

\author{
Roi Treister ${ }^{1,2}$ \\ Thomas A Eaton ${ }^{3}$ \\ Jeremiah J Trudeau ${ }^{4}$ \\ Harrison Elder ${ }^{2}$ \\ Nathaniel P Katz ${ }^{2,5}$ \\ 'Department of Neurology, \\ Massachusetts General Hospital, \\ Harvard Medical School, Boston, \\ ${ }^{2}$ Analgesic Solutions, Natick, \\ MA, ${ }^{3}$ Department of Psychology, \\ University of Connecticut, Storrs, \\ ${ }^{4}$ Boehringer Ingelheim, Ridgefield, CT, \\ ${ }^{5}$ Anesthesiology and Perioperative \\ Medicine, Tufts University School of \\ Medicine, Boston, MA, USA
}

\begin{abstract}
Clinical trials of analgesics have been plagued with poor assay sensitivity due, in part, to variability in subjects' pain reporting. Herein, we develop and evaluate the focused analgesia selection test (FAST), a method to measure patients' pain reporting skills. Subjects with osteoarthritis of the hip, knee, and/or ankle with pain intensity of $\geq 3 / 10$ on a $0-10$ numerical rating scale were enrolled. Subjects underwent the FAST procedure, which consists of recording subjects' pain reports in response to repeated administration of thermal noxious stimuli of various intensities applied on the arm with the Medoc ${ }^{\circledR}$ Thermal Sensory Analyzer II. Subjects also rated non-noxious stimuli consisting of visual contrast rating. After performing an exercise task, subjects also rated clinical pain and were asked to report whether their pain had increased, decreased, or stayed the same. Overall, 88 subjects were enrolled, and 83 were included in the analyses. FAST's outcomes including the $R^{2}$, intraclass correlation coefficient (ICC), and coefficient of variation $(\mathrm{CoV})$ indicated that subjects' pain reporting skills were widely distributed. Higher FAST ICC significantly predicted greater changes in clinical pain following exercise ( $p=0.017$ ), whereas the visual contrast test did not predict postexercise pain. FAST is the first method that measures subjects' pain reporting skills. Using FAST to enrich clinical trials with "good" pain reporters (with high FAST ICC) could increase assay sensitivity. Further evaluation of FAST is ongoing.
\end{abstract}

Keywords: pain assessment, pain variability, analgesic clinical trials, pain psychophysics

\section{Introduction}

The failure of clinical trials of effective analgesic medications observed in the past decades has been termed "low assay sensitivity", which refers to issues in the design or conduct of clinical trials that diminish the ability of such trials to discriminate between effective treatment and placebo. ${ }^{1}$

To discriminate between an effective analgesic compound and placebo, a clinical study requires adequate statistical power. Calculations of statistical power involve 2 essential components: treatment group differences (difference in mean pain scores between study arms) and variation of those pain scores., ${ }^{2,3}$ A substantial body of research has explored practices and procedures to maximize treatment group differences, mainly by focusing efforts on optimizing study designs and outcome measures. ${ }^{1,4,5}$

Variation in pain reports has not undergone similar rigorous examination. Research has suggested that patients with inconsistent pain reports continue to have inconsistent pain reports over time, ${ }^{6}$ and that individuals with large pain intensity variations are more likely to respond to placebo ${ }^{7}$ or respond well to both the analgesic and placebo. ${ }^{8}$ Most recently, a meta-analysis of 12 clinical trials in postherpetic neuralgia and painful
Analgesic Solutions, 232 Pond St, Natick, MA 0I760, USA

$\mathrm{Tel}+\mathrm{I} 78 \mid 4449605$

Fax +l 5086529099

Email NKatz@analgesicsolutions.com 
diabetic peripheral neuropathy demonstrated that variability in pain reports during the baseline week was associated with increased likelihood of response in the placebo-treated group. ${ }^{9}$ The authors suggested that excluding patients with a high variability in their baseline 7-day pain scores has the potential to improve assay sensitivity of analgesic clinical trials.

Variability in pain scores, as in most other measures, consists of 2 components: true variance and error variance. True variance refers to the actual fluctuations in the underlying construct. Pain does actually vary from day to day and even hour to hour in many pain patients. Superimposed upon this true variance is error variance, which refers to variability due to any source of error in the measurement of the underlying construct. For example, if a patient's pain is truly $4 / 10$ two days ago, $5 / 10$ yesterday, and 6/10 today, the mean would be 5 and the standard deviation (SD) 1.0. Compare that patient to another patient who experiencing the same pain, but is less skilled in reporting his pain accurately: for instance, he reports $2 / 10$ two days ago, $6 / 10$ yesterday, and $8 / 10$ today. The mean would be 5.3 and the SD of 3.06. The greater the error variance, the larger the sample size needed to differentiate 2 groups, the smaller the statistical power, and the greater the study's bias to detect no difference.

Some patients may report their pain levels reliably (i.e., reporting the same pain intensity in response to a specific stimulus) and accurately (i.e., in good approximation to the "real" experience) while others' pain reports may vary widely for the same experience or may not be good approximation of the real experience. Our hypothesis is that subjects differ in their pain reporting skills, and that selecting study subjects with good pain reporting skills should improve assay sensitivity in analgesic trials. To this end, we developed and initially tested the focused analgesia selection test (FAST), a method that was intended to measure patients' pain reporting skills. FAST uses an experimental pain paradigm that consists of exposing subjects to multiple painful stimuli of different intensities in a blinded fashion to quantify pain reporting reliability and accuracy. We hypothesized that FAST can discriminate between subjects with good versus poor pain reporting skills, and that pain reporting skills, as measured by FAST, would predict the ability to accurately report changes in clinical pain.

\section{Materials and methods Subjects}

Subjects with chronic osteoarthritis (OA) pain were recruited. All participants signed an informed consent, and the study was approved by an Institutional Review Board (Asentral
IRB). Subjects aged $\geq 21$ years were eligible to participate in the study if they had OA of the hip, knee, and/or ankle (by medical history), had experienced OA pain for at least 6 months, and had a pain intensity of at least $3 / 10$ on a $0-10$ numerical rating scale (NRS) (where 0 means "no pain" and 10 means "worst pain imaginable") for the past 15 days. Subjects who were pregnant or breastfeeding, who had any other chronic pain syndrome (e.g., fibromyalgia, rheumatoid arthritis, uncontrolled inflammatory arthritis, or nonsteroidal anti-inflammatory drug [NSAID]-dependent inflammatory arthritis), or had a condition that prevented them from adequately completing the procedures were excluded from the study.

\section{The FAST}

FAST is based on recording a subject's pain reports in response to repeated administration of thermal noxious stimuli of various intensities to assess subject's pain reporting skills. Specifically, FAST utilizes the MEDOC TSA II device. Thermal Sensory Analyzer II that incorporates a Peltier element-based thermode $\left(30 \times 30 \mathrm{~mm}^{2}\right)$. The thermode was applied to the ventral surface of the subject's nondominant arm and the temperature was raised from a baseline of $32^{\circ} \mathrm{C}$, peaked for 3 seconds at 1 of 7 designated temperatures $\left(43^{\circ} \mathrm{C}, 45^{\circ} \mathrm{C}, 47^{\circ} \mathrm{C}, 48^{\circ} \mathrm{C}, 49^{\circ} \mathrm{C}, 50^{\circ} \mathrm{C}\right.$, or $\left.51^{\circ} \mathrm{C}\right)$, and then decreased down to the baseline. Stimulus duration was always 8 seconds, meaning that the rate of rise and fall varied from 3.3 to $9^{\circ} \mathrm{C} /$ seconds depending on the destination temperature. Subjects were asked to rate the peak pain intensity of each stimulus by using a computerized visual analog scale (CoVAS): a box attached to the computer with a (0-100 mm) slider that moves along a line from "no pain" to "worst pain imaginable". Each temperature was presented 7 times in a random block-ordered design (total of 49 stimuli). Stimuli were triggered manually, allowing the subject sufficient time to rate the pain they perceived during each stimulus, resulting in interstimulus intervals of $10-20$ seconds. The location of the thermode was adjusted every 14 stimuli to minimize sensitization and/or habituation effects. The duration of the FAST procedure is $\sim 25$ minutes.

\section{Nonnoxious stimulus rating (visual contrast rating test)}

To evaluate whether the FAST results are a specific characterization of subject's ability to accurately rate their pain, as opposed to a nonspecific measure of the ability to use rating scales, in general, a visual contrast rating test was used. In 
this procedure, subjects were presented stimuli that consisted of 1 of 7 black/white contrasts on a tablet computer monitor. Each contrast stimulus consisted of a square (whose color ranged from white, through various shades of gray, to black) on a white background. The stimulus ranged from a $0 \%$ contrast (i.e., white square on a white background) to a $100 \%$ contrast stimulus (i.e., black square on a white background). As with FAST, each stimulus was presented 7 times, resulting in 49 total stimuli. The subject's task was to evaluate the stimuli in terms of the degree of perceived contrast by rating the stimuli on a visualized analog scale (VAS) from "no contrast" to "extreme contrast". Duration of the visual contrast rating test was $\sim 5$ minutes.

\section{Clinical pain rating}

Participants were asked to perform a standardized exercise task involving their affected joint consisting of walking up and down a flight of 15 stairs (7.5 inch each) twice (a total of 30 stairs up and down). Subjects rated their pain on a 0-100 mm VAS before and after exercise. After exercise, subjects were also asked if their pain had increased, decreased, or stayed the same as a result of the exercise. Subjects who reported an increase in pain after exercise were considered “exercise responders".

\section{Study design}

Subjects were asked to refrain from taking any as-needed analgesics for 12 hours prior to their study visit. Those subjects on fixed doses of analgesics continued to take them. After signing the informed consent, subjects participated in familiarization session to acquaint themselves with FAST and the visual contrast rating task procedures, followed by the actual procedures. After completion of these 2 procedures, all subjects underwent the exercise task.

In addition, a battery of 13 surveys or questionnaires that measure various psychosocial factors and states were completed by all subjects to assess whether psychological characteristics correlate with FAST results and if any of these characteristics could be used alongside FAST to improve prediction of subject's ability to accurately report their clinical pain. This battery included the Brief Pain Inventory-Short Form, McGill Pain Questionnaire-Short Form, Center for Epidemiological Studies Depression Scale, State-Trait Anxiety Inventory for Adults, Eysenck Personality Questionnaire-Abbreviated, PHQ-15, Pain Catastrophizing Scale, Kohn Reactivity Scale, Fear of Pain Questionnaire-III, Pain Attitudes Questionnaire-Revised,
Expectation for Pain Relief from Treatment, Hopefulness for Pain Relief with Treatment, Nottingham Health Profile, Social Desirability Scale, and the Locus of Control Scale. Given that the FAST results did not improve significantly from the addition of these characteristics, these results are not presented in the current manuscript. All procedures were completed in $\sim 3$ hours, and participants were paid $\$ 150$ for their participation.

\section{Statistical analyses}

Analyses were conducted using Statistical Package for the Social Sciences (SPSS) for Windows version 19 (SPSS, Inc., Chicago, IL, USA). Descriptive statistics were used to present demographic and baseline characteristics.

As most variables were nonnormally distributed (tested by Kolmogorov-Smirnov and Shapiro-Wilk tests), data were mostly analyzed with nonparametric tests. Friedman's tests (followed by Wilcoxon post hoc test, when applicable) were used to assess possible order effects in pain scores in response to the thermal stimuli. Spearman's correlations were used to assess relations between the 3 outcomes $\left(R^{2}\right.$, intraclass correlation coefficient [ICC], and coefficient of variation $[\mathrm{CoV}])$, both in the FAST and the visual contrast rating test. The variable "change in clinical pain" (before and after the exercise) was normally distributed ( $p=0.85)$; hence, regression was used to predict changes in clinical pain. Pain scores captured during the FAST procedure were used to calculate 3 FAST outcomes as follows: 1) $R^{2}$ was calculated by using a power model regression. Disparity between the predicted function and actual scores could be a result of inaccuracy or unreliability. Close concordance between actual and predicted scores (higher $R^{2}$ ) suggests greater accuracy and reliability. 2) ICC was computed using a 2-way mixed model for the 7 presentations of each of the 7 intensity levels. An ICC score approaching 1.0 denotes a high degree of reliability, or the agreement in responses to the same stimulus over several presentations. 3) The CoV is the ratio of the $\mathrm{SD}$ to the mean. The average $\mathrm{CoV}$ was calculated as the mean of $7 \mathrm{CoVs}, 1$ at each stimulus level. A higher $\mathrm{CoV}$ demonstrates a larger variability in reporting. Pearson's correlations were used to assess relationships between the FAST outcomes. To assess whether the FAST outcomes predict change in pain following exercise, regression analyses were conducted with the FAST outcomes as predictors (separately in repeated regressions) and change in VAS as dependent variable. In all figures, data presented as mean $\pm \mathrm{SD}$ unless specified otherwise. 


\section{Results}

\section{Subject demographic and baseline characteristics}

The total study population consisted of 88 subjects. Of the 88 study subjects, 5 were excluded from analysis because of $>20 \%$ missing values during the FAST procedure. Thus, the data analysis consisted of 83 subjects. Subjects ( 38 males; 45 females) had a mean age of $60.4 \pm 7.5$ years; $54 \%$ were White, $24 \%$ were Black, and the remaining subjects were of "other" races. Subjects were taking NSAIDs $(\mathrm{n}=55)$, acetaminophen $(n=32)$, opioids $(n=16)$, COX-2 inhibitors $(n=4)$, aspirin $(n=6)$, and gabapentin/pregabalin $(n=3)$ for their OA pain (few subjects $(\mathrm{n}=16)$ took $>1$ medication class). Subjects' baseline pain scores on 0-10 NRS were as follows: least amount of pain in the last 24 hours: $4.87 \pm 2.71$, worst amount of pain in the last 24 hours: $6.23 \pm 2.02$, average pain level: $5.47 \pm 2.08$, and level of pain right now: $4.82 \pm 2.45$.

\section{Pain intensity in response to the FAST algorithm}

Mean pain intensities reported in response to each of the 7 stimuli intensities are presented in Figure 1. Pain intensities induced by the FAST algorithm spanned the entire pain scale spectrum from 0 to 100 . Group mean \pm SD (median) responses ranged from $4.9 \pm 8.5$ (1.5) for the lowest stimulus intensity $\left(43^{\circ} \mathrm{C}\right)$ to $72.3 \pm 24.9$ (79.9) for the highest stimulus $\left(51^{\circ} \mathrm{C}\right)$. Median pain scores significantly differed from each other (Friedman's test, chi-square 426.6; $p$ value $<0.001$ followed by post hoc Wilcoxon tests, all $p$ values $<0.001$ ).

Mean pain intensities in response to each stimulus are presented in Figure 2. For each stimulus intensity, the 7 bars represent the 7 repetitions of stimuli for each intensity, organized by order (for each stimulus intensity, the left bar represents the

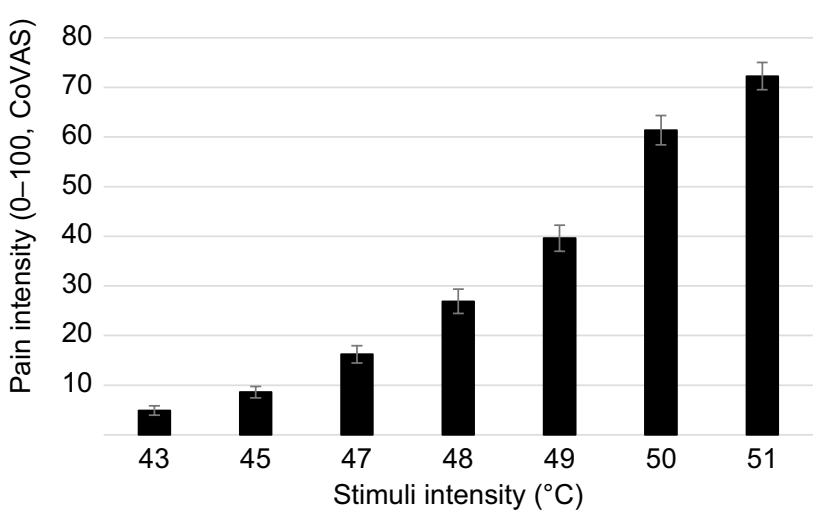

Figure I Mean pain scores in response to the 7 focused analgesia selection test stimuli by stimulus intensity.

Notes: Black bars represent the average pain scores in response to the 7 stimuli at each intensity. Error bars represent the standard error of the mean.

Abbreviation: CoVAS, computerized visual analog scale. first stimulus and the right bar represents the 7 th stimulus). For lower stimuli intensities $\left(43^{\circ} \mathrm{C}, 45^{\circ} \mathrm{C}\right.$, and $\left.47^{\circ} \mathrm{C}\right)$, there was a small yet statistically significant order effect: pain reports slightly decreased over time, representing a possible habituation effect (Friedman's tests, $p=0.02, p<0.001$, and $p=0.041$, respectively). In the highest stimulus intensity $51^{\circ} \mathrm{C}$, median pain scores significantly increased, $(p=0.001)$ implying on a sensitization effect. No other order effect was found in the other stimuli intensities (Freidman's tests, $p=0.873, p=0.544$, $p=0.490$, for $48^{\circ} \mathrm{C}, 49^{\circ} \mathrm{C}$, and $50^{\circ} \mathrm{C}$, respectively).

To assess overall order effects, pain intensities in response to each stimulus order (1st, 2 nd, etc.) were averaged across stimulus intensities (i.e., average pain scores of the first stimulus of each intensity, average pain scores of the 2 nd stimulus of each intensity, etc., Figure 3). No significant difference was found between the mean pain intensities of stimulus sequence scores (Freidman's test, $p=0.095$ ).

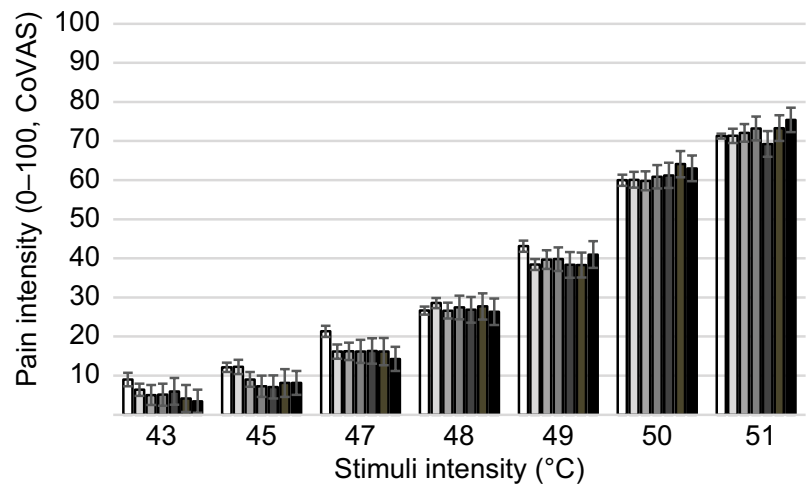

Figure 2 Mean pain scores in response to the 49 focused analgesia selection test stimuli by stimulus order.

Notes: For each stimulus intensity, the 7 bars represent the 7 repetitions of stimuli for each intensity, organized by order. The white bar on the left represents the first stimulus and the black bar on the right the 7th stimulus. Error bars represent the standard error of the mean.

Abbreviation: CoVAS, computerized visual analog scale.

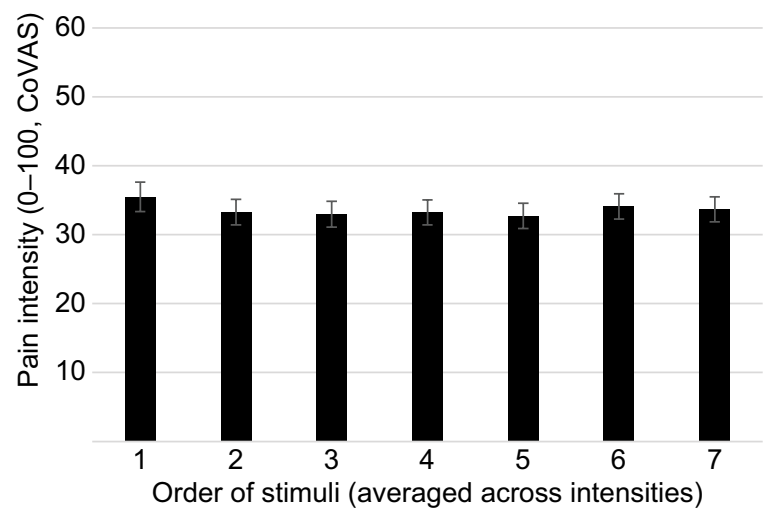

Figure 3 Mean pain scores in response to the 7 focused analgesia selection test stimuli by stimulus order.

Notes: Black bars represent the average pain scores in response to each stimulus order, across stimulus intensities. Error bars represent the standard error of the mean.

Abbreviation: CoVAS, computerized visual analog scale. 


\section{FAST outcomes}

Descriptive statistics of the FAST outcomes are described in Table 1 . The $R^{2}$, ICC, and $\mathrm{CoV}$ indicated that subjects' pain reporting skills were widely distributed. Overall, $70 \%$ of the subjects had an ICC value $>0.70$ (range $0.09-0.95$ ), indicating good reliability. $R^{2}$ had a mean of 0.61 (range $0.15-0.89$ ), whereas $\mathrm{CoV}$ had a mean of 0.74 (range $0.23-1.58$ ). Subjects with a high $R^{2}$ had high ICC values (Spearman's $r=0.635$, $p<0.001$ ) and low CoV values (Spearman's $r=-0.425, p<0.001$ ). Therefore, these 3 measures seemed to be internally consistent.

Figure 4 illustrates the FAST results of 2 representative subjects. The results of a subject who demonstrated low variation in his pain reports ("good" pain reporter) are illustrated in Figure 4A ( $\left.\mathrm{CoV}=0.42, \mathrm{ICC}=0.91, R^{2}=0.72\right)$. Figure 4B illustrates the results of a subject who demonstrated high variability in his pain reports ("poor" pain reporter; $\mathrm{CoV}=0.76$, ICC $=0.58, R^{2}=0.47$ ). In these figures, each $\mathrm{X}$ represents 1 rating of 49 stimuli administrated at 7 intensities.

Table I FAST outcomes

\begin{tabular}{llll}
\hline & $\boldsymbol{R}^{2}$ & ICC & CoV \\
\hline Mean (SD) & $0.606(0.16)$ & $0.782(0.16)$ & $0.740(0.3 \mathrm{I})$ \\
Median & 0.64 & 0.82 & 0.70 \\
Minimum & 0.15 & 0.09 & 0.23 \\
Maximum & 0.89 & 0.95 & 1.58
\end{tabular}

Abbreviations: CoV, coefficient of variation; FAST, focused analgesia selection test; ICC, intraclass correlation coefficient; SD, standard deviation.

\section{Visual contrast rating test}

Subjects accurately perceived and rated the contrast stimuli and used the full range of the VAS scale (data not shown). Mean \pm SD ICC values were $0.98 \pm 0.05$ (range 0.58-1.00; median 0.99), indicating good reliability. $R^{2}$ had a mean of $0.79 \pm 0.16$ (range 0.09-0.94; median 0.84), and CoV had a mean of 0.30 \pm 0.17 (range 0.01-0.72; median 0.27). These results indicating that subjects reliably reported the same response for each stimulus level across presentations. Like the FAST results, all measures of visual contrast report reliability $\left(R^{2}, \mathrm{ICC}\right.$, and $\left.\mathrm{CoV}\right)$ were highly intercorrelated, indicating convergent validity. However, none of these measures of visual contrast report were correlated with the FAST outcomes, nor with any clinical pain measure.

\section{Clinical pain}

After the exercise task, 57 subjects (68\%) reported that their pain had increased after exercise and so were considered "exercise responders". However, even within this subgroup, postexercise pain scores (5.16 \pm 2.94 ; median 5.0) were not significantly higher than pre-exercise pain scores $(4.75 \pm 2.45$; median 5.08; Wilcoxon's test, $p=0.330$ ).

\section{FAST results predicted change in clinical pain}

In the exercise responders subgroup, the FAST ICC significantly predicted changes in clinical pain following exercise

A

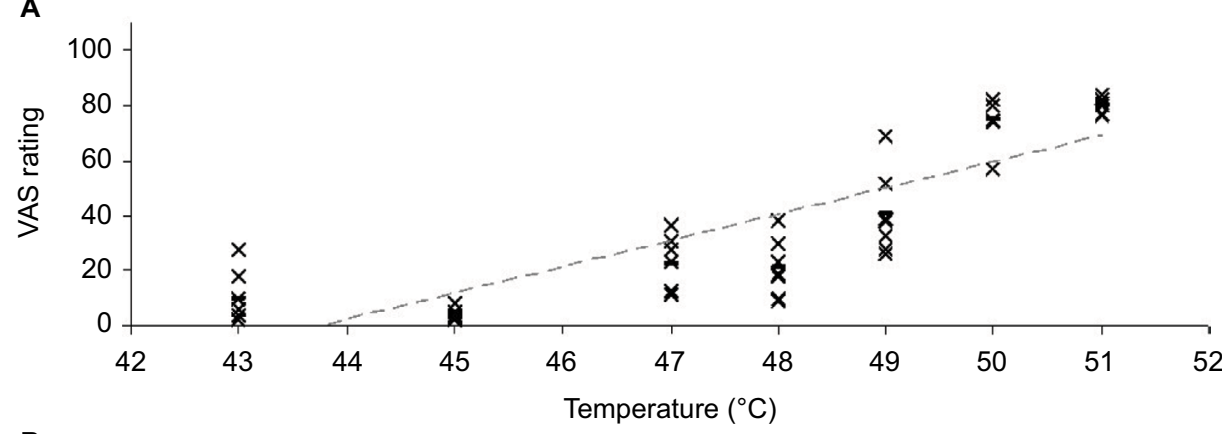

B

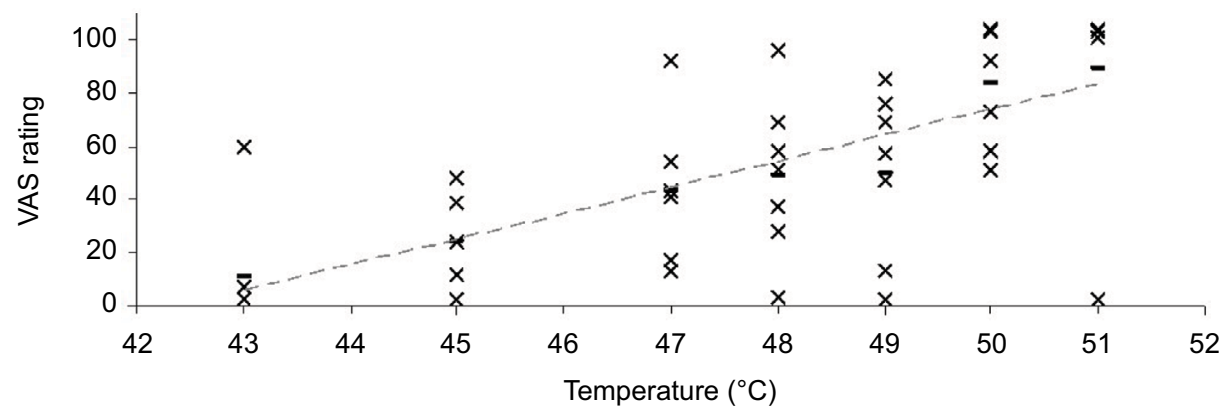

Figure 4 Examples of FAST results obtained for a "good" vs a "poor" pain reporter.

Notes: (A) Illustrates the FAST results of a "good" pain reporter. (B) Illustrates the FAST results of a "poor" pain reporter. Each X represents I rating of 49 stimuli administrated at 7 intensities.

Abbreviations: FAST, focused analgesia selection test; VAS, visualized analog scale. 
2.00

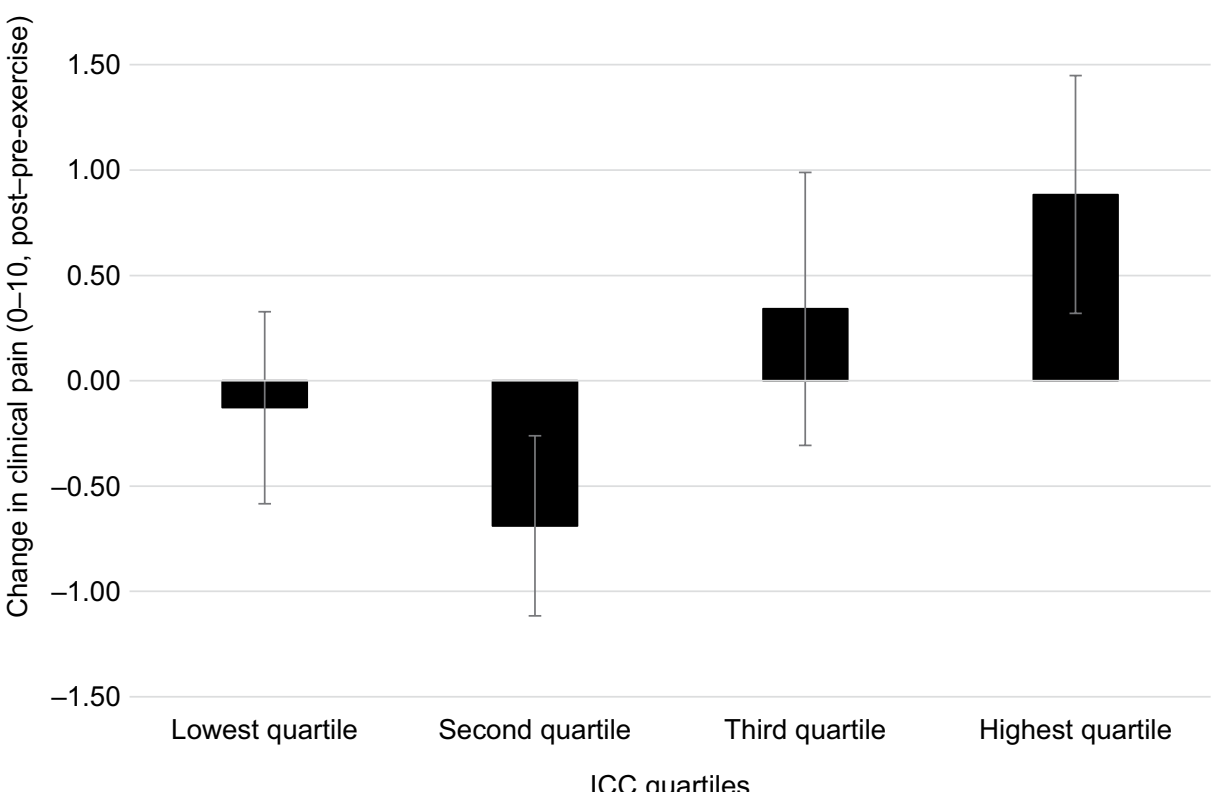

ICC quartiles

Figure 5 Changes in clinical pain following exercise by FAST ICC quartiles.

Notes: Changes in clinical pain before and after the exercise for each of the cohort quartiles, as defined by their FAST ICC scores. The first bar from the left represents the $25 \%$ of the cohort that were the least accurate (lowest ICC score). The bar from the right, the highest quartile, represents the $25 \%$ of the cohort that the most accurate in the FAST procedure.

Abbreviations: FAST, focused analgesia selection test; ICC, intraclass correlation coefficient.

(Beta $=0.327, F=6.107, p=0.017)$, unlike the $R^{2}(p=0.206)$ and $\mathrm{CoV}(p=0.686)$. Changes in clinical pain were the largest in subjects with best ICC (Figure 5, presented as 4 groups, based on ICC quartiles). Similar results were found in the entire cohort with ICC being a significant predictor of changes in clinical pain following exercise (Beta $=0.274, F=5.774$, $p=0.019)$, but not the $R^{2}(p=0.716)$ or $\operatorname{CoV}(p=0.439)$. The parameters of the visual contrast-rating test did not predict change in pain after exercise in the entire cohort or the exercise responders subgroup. To assess if the subjects' characteristics are related to their ability to accurately report their pain, we divided the cohort into 2 equal groups by ICC median ("poor" and "good" pain reporters) and compared demographics, baseline pain data, and medication consumption between the groups. No significant differences were found in any of these characteristics between the good and poor pain reporters.

\section{Discussion}

The aim of the current study was to evaluate FAST as a potential method to measure patients' pain reporting skills. As hypothesized, subjects demonstrated a wide range of pain reporting skills as assessed by FAST outcomes, and among these the ICC, a measure of reliability, predicted changes in clinical pain following exercise.
The main finding of the present study is that the FAST ICC significantly predicted the change in a subject's clinical pain following exercise. Climbing staircases should increase OA pain, and a better pain reporter should report it more accurately. The higher the ICC score is, the lower the variability in pain reports (i.e., high reliability) and the better the subjects pain reporting skills. The positive correlation between the FAST ICC and the change in clinical pain following exercise implies that those who demonstrated greater pain reporting skills (higher ICC) reported greater increases in their clinical pain following exercise. The result suggests that by using FAST one can identify subjects who can more accurately report changes in clinical pain.

The fact that the FAST outcome measures $\left(R^{2}\right.$, ICC, and $\mathrm{CoV})$ demonstrated strong correlations among each other suggests that these measures do quantify-related aspects of pain reporting skills. Moreover, the fact that the $R^{2}$, ICC, and $\mathrm{CoV}$ calculated from the visual contrast rating test were not correlated with those calculated with FAST, nor with the change in clinical pain, suggests that FAST results are pain specific, rather than an assessment of general ability to use response scales.

Another central finding is that FAST was feasible, welltolerated, and was not influenced by robust habituation or 
sensitization effects that could potentially jeopardize its ability to accurately quantify subjects' pain reporting skills. Our working hypothesis is that a significant component of the variability in reporting pain in response to sequential stimuli of the same intensity is due to error in pain reporting, rather than true change in the painfulness of the stimuli due to either habituation or sensitization. In other words, similar pain intensity should be perceived (and reported) in response to repeated exposure to noxious stimuli of fixed intensities, and inconsistencies in pain scores are due to inaccurate reports. This assumption depends on minimizing other processes that might alter the pain perceived in response to an experimental stimulus. For this reason, we carefully assessed the possible effects of stimuli order, since habituation (decrease in pain over time) or sensitization (increase in pain over time) is probable, especially when exposing subjects to multiple noxious stimuli. Our results revealed that overall there was no significant order effect except at lower stimulus intensities $\left(43^{\circ} \mathrm{C}\right.$ and $\left.45^{\circ} \mathrm{C}\right)$, where a small - yet significant - habituation effect was observed. This suggests that the variability in pain scores in response to these stimuli may contain a true variance component, meaning that FAST outcomes may not be a "pure" reflection of pain reporting skills (i.e., subjects accurately reported different pain intensities in response to the $43^{\circ} \mathrm{C}$ and $45^{\circ} \mathrm{C}$ stimuli not because they are poor responders but because of habituation). To decrease the possibility of habituation or sensitization, the FAST algorithm used herein included interstimulus intervals of 10-20 seconds, and the thermode position was slightly adjusted every 14 stimuli. Habituation and/or sensitization could be further minimized by using longer interstimulus intervals and by moving the thermode more frequently. Nonetheless, we believe that FAST outcomes will prove useful even though some "noise" remains.

Worth notice is that the increase in pain intensity following exercise was not significant, even in the subgroup of patients who reported that their pain was increased following exercise. Nonetheless, the FAST ICC did correlate with the small change in clinical pain induced by the exercise.

Application of quantitative sensory testing in pain research (QST, e.g., pain psychophysics) is traditionally performed to characterize subjects' pain sensitivity profile, to assess the effects of various manipulations/treatments on experimentally induced pain, and to learn about pain's underlining mechanisms. As far as we know, the FAST approach is the first utilization of QST outside its abovementioned common objectives. Additional studies are needed to further evaluate FAST's ability to assess subjects' pain reporting skills and its clinical relevance. This includes confirming that FAST outcomes correlate with patient's variability in clinical pain scores and determining FAST psychometric properties, such as test-retest reliability. The most direct evidence of FAST's ability to assess patient's pain reporting skills will be to use it at baseline, prior to randomization into a placebo controlled trial. This will allow researchers to determine whether subjects with better pain reporting skills have higher effect sizes in response to treatment. These studies are currently on going.

Importantly, it is yet to be proven that the FAST results reflect pain reporting accuracy. Another explanation is that the between subject variation in FAST outcomes might reflect subject's tendency to report their pain differently in response to the same stimuli. In other words, some subjects might be more vulnerable than others to physiological processes or environmental cues that modulate pain, hence their poor performance in the FAST.

Few limitations deserve consideration. First, the cohort included heterogeneous subjects with OA of the hip, knee, and/or ankle. Depending on the affected joint, the subjects might respond differently to exercise. Second, the QST device used in the current study might not be most suitable for use in a multicenter clinical trial setting. To address this issue, a different, more cost-effective, and user-friendly QST device is being used in ongoing FAST studies. Third, the current study results FAST predicted changes in clinical pain following exercise. The exercise was performed as an intervention aimed to induce changes in pain (i.e., to increase pain), and our assumption is that FAST will demonstrate similar predictive properties when changes in clinical pain are due to analgesic intervention aimed to reduce pain in clinical trial. This assumption is yet to be proven. Lastly, 5 subjects $(6 \%)$ were excluded from analysis due to missing values in the FAST procedure due to intolerability to the higher range of stimuli. In ongoing studies, the FAST consists of slightly lower stimuli intensities.

To the best of our knowledge, the FAST procedure presented herein is the only procedure intended to assess a subject's ability to accurately and reliably report pain. By using FAST one can distinguish between "good" and "poor" pain reporters. Excluding "poor" reporters from clinical trials - and therefore enriching trials with "good" pain reporters - might increase signal to noise ratios in clinical trials, thus diminishing the potential for failed clinical trials 
of effective treatments. Further research is needed to confirm our results and to further validate the FAST in other chronic pain populations.

\section{Acknowledgment}

The authors wish to thank Pfizer, Inc. for funding this project.

\section{Disclosure}

NPK is the CEO of Analgesic Solutions, a clinical research and consulting firm with many clients throughout the pharmaceutical industry. All other authors are employees or former employees of Analgesic Solutions.

\section{References}

1. Dworkin RH, Turk DC, Peirce-Sandner S, et al. Research design considerations for confirmatory chronic pain clinical trials: IMMPACT recommendations. Pain. 2010;149(2):177-193.
2. Houle TT, Penzien DB, Houle CK. Statistical power and sample size estimation for headache research: an overview and power calculation tools. Headache. 2005;45(5):414-418.

3. Abdulatif M, Mukhtar A, Obayah G. Pitfalls in reporting sample size calculation in randomized controlled trials published in leading anaesthesia journals: a systematic review. Br J Anaesth. 2015;115(5):699-707.

4. Katz N. Methodological issues in clinical trials of opioids for chronic pain. Neurology. 2005;65(12 Suppl 4):S32-S49.

5. Katz J, Finnerup NB, Dworkin RH. Clinical trial outcome in neuropathic pain: relationship to study characteristics. Neurology. 2008;70(4):263-272.

6. Quiton RL, Greenspan JD. Across- and within-session variability of ratings of painful contact heat stimuli. Pain. 2008;137(2):245-256.

7. Harris RE, Williams DA, McLean SA, et al. Characterization and consequences of pain variability in individuals with fibromyalgia. Arthritis Rheum. 2005;52(11):3670-3674.

8. Palmer RH, Turk DC, Hufford MR, Wang Y. The impact of pain variability on response to milnacipran and placebo in two trials of patients with fibromyalgia. Presented at: The 13th World Congress on Pain; August; 2010; Montreal, Canada.

9. Farrar JT, Troxel AB, Haynes K, et al. Effect of variability in the 7-day baseline pain diary on the assay sensitivity of neuropathic pain randomized clinical trials: an ACTTION study. Pain. 2014;155(8):1622-1631.
Journal of Pain Research

\section{Publish your work in this journal}

The Journal of Pain Research is an international, peer reviewed, open access, online journal that welcomes laboratory and clinical findings in the fields of pain research and the prevention and management of pain. Original research, reviews, symposium reports, hypothesis formation and commentaries are all considered for publication.

\section{Dovepress}

The manuscript management system is completely online and includes a very quick and fair peer-review system, which is all easy to use. Visit http://www.dovepress.com/testimonials.php to read real quotes from published authors. 